\title{
Hepatic Inflammatory Myofibroblastic Tumor Detected in the Fetal Period That Caused an Oncologic Emergency
}

\author{
Hirotaka Kato $^{a}$ Yasuyuki Mitani ${ }^{a}$ Taro Goda $^{a}$ Masaki Ueno ${ }^{a}$ \\ Shinya Hayami $^{a}$ Hiroshi Tsujimoto $^{b}$ Shinji Kounami ${ }^{b}$ Takayuki Ichikawa ${ }^{b}$ \\ Hiroki Yamaue ${ }^{a}$ \\ aSecond Department of Surgery, Wakayama Medical University, Wakayama, Japan; \\ ${ }^{b}$ Department of Pediatrics, Wakayama Medical University, Wakayama, Japan
}

Keywords

Inflammatory myofibroblastic tumor $\cdot$ Liver $\cdot$ Neonate $\cdot$ Oncologic emergency $\cdot$ Fetal diagnosis

\begin{abstract}
A huge abdominal cystic lesion with ascites was detected in a male neonate at 31 weeks of gestation. Increasing ascites and the appearance of subcutaneous edema were detected, which caused fetal hydrops. The patient was delivered by emergency cesarean section at 33 weeks of gestation. The birth weight was $2,407 \mathrm{~g}$, and the Apgar score was 8/9 points (1-/5min values). Breathing at birth was stable, but the patient presented with remarkable abdominal distention due to the ascites. Later, the patient presented with tachypnea, and breathing gradually worsened, so an emergency operation was performed. There were no intraoperative findings within the small intestine, but there was a large amount of ascites and a cystic mass arising from the liver. The patient's breathing and circulation dynamics could only be stabilized by ascites removal, so only a tumor biopsy was performed. The pathological findings led to the diagnosis of an inflammatory myofibroblastic tumor, and steroids were administered early after surgery for the purpose of an anti-inflammatory effect and tumor shrinkage. The abdominal distention was alleviated, and blood examinations showed a reduced inflammatory response. There was no apparent shrinkage of the tumor, however; thus, radical surgical treatment was performed on day 24. The postoperative course was uneventful, so the patient was discharged on day 36. Seven years after the operation there has been no recurrence or distant metastasis.




\section{Case Reports in Oncology}

\begin{tabular}{l|l}
\hline Case Rep Oncol 2020;13:1513-1519 \\
\hline DOI: 10.1159/000511052 & $\begin{array}{l}\text { @ 2020 The Author(s). Published by S. Karger AG, Basel } \\
\text { www.karger.com/cro }\end{array}$ \\
\hline
\end{tabular}

Kato et al.: Oncologic Emergency in Neonatal Hepatic Inflammatory Myofibroblastic Tumor

\section{Introduction}

Inflammatory myofibroblastic tumors (IMTs) are solid tumors showing proliferation of the collagen matrix and spindle-shaped cells with proliferation of myofibroblasts and infiltration of inflammatory cells such as lymphocytes, plasma cells, and eosinophils. The etiology of hepatic IMT has not been fully understood.

This is the first case report of hepatic IMT in the fetal period. Huge hepatic IMTs can cause an oncologic emergency in the neonatal period due to respiratory disorder associated with ascites. Immediate action is therefore necessary to save these patients.

\section{Case Presentation}

Fetal ultrasonography at 31 weeks and 5 days of gestation revealed a cystic lesion $(70 \times$ $47 \times 55 \mathrm{~mm}$ ) with ascites (Fig. 1). Fetal ultrasonography at 33 weeks and 5 days of gestation revealed increased ascites and the appearance of subcutaneous edema, which caused fetal hydrops. The patient was therefore delivered by emergency cesarean section. The birth weight was 2,407 g, and the Apgar score was $8 / 9$ points (1-/5-min values).

The patient's mother was naturally healthy and had no underlying diseases. Hepatitis B, hepatitis C, TPHA, HIV, HTLV-1, and chlamydia were all negative. The value for rubella was $1: 64$, and that for toxoplasma was $<1: 16$.

At birth, the patient's pulse rate was $178 \mathrm{bpm}, \mathrm{SpO}_{2}$ was $100 \%$, the respiratory rate was 50 breaths/min, blood pressure was $50 / 26 \mathrm{~mm} \mathrm{Hg}$, and there was remarkable abdominal distention.

Blood gas analysis and biochemical examination at birth showed the following results: pH 7.266, $\mathrm{PaCO}_{2} 40.2 \mathrm{~mm} \mathrm{Hg}, \mathrm{PaO}_{2} 56.3 \mathrm{~mm} \mathrm{Hg}, \mathrm{HCO}_{3} 24.8 \mathrm{mmol} / \mathrm{L}, \mathrm{BE}-2.7 \mathrm{mmol} / \mathrm{L}$, Lac 16 $\mathrm{mg} / \mathrm{dL}, \mathrm{WBC} 10,200 / \mu \mathrm{L}, \mathrm{RBC} 3,750,000 / \mu \mathrm{L}, \mathrm{Hb} 13.9 \mathrm{~g} / \mathrm{dL}, \mathrm{Ht} 42.7 \%$, PLT 347,000/ $\mathrm{LL}$, TP 3.9 mg/dL, Alb 1.8 g/dL, CK 41 IU/L, AST 104 IU/L, ALT 6 IU/L, LDH 1,234 IU/L, Cre 0.48 mg/dL, UA $4.6 \mathrm{mg} / \mathrm{dL}$, BUN $9 \mathrm{mg} / \mathrm{dL}$, Na $138 \mathrm{mEq} / \mathrm{L}, \mathrm{K} 6.8 \mathrm{mEq} / \mathrm{L}, \mathrm{Cl} 105 \mathrm{mEq} / \mathrm{L}, \mathrm{Mg} 2.2 \mathrm{mg} / \mathrm{dL}$, Ca $9.7 \mathrm{mg} / \mathrm{dL}$, IP $6.0 \mathrm{mg} / \mathrm{dL}$, T-Bil $1.4 \mathrm{mg} / \mathrm{dL}$, D-Bil $0.0 \mathrm{mg} / \mathrm{dL}$, and CRP $6.34 \mathrm{mg} / \mathrm{dL}$. A strong inflammatory response was shown.

Chest-abdominal plain radiography showed diaphragm elevation and chest compression. Abdominal ultrasonography showed cystic lesions across the entire abdomen. Immediately after birth, the patient had no tachypnea, but it gradually developed. Blood gas analysis

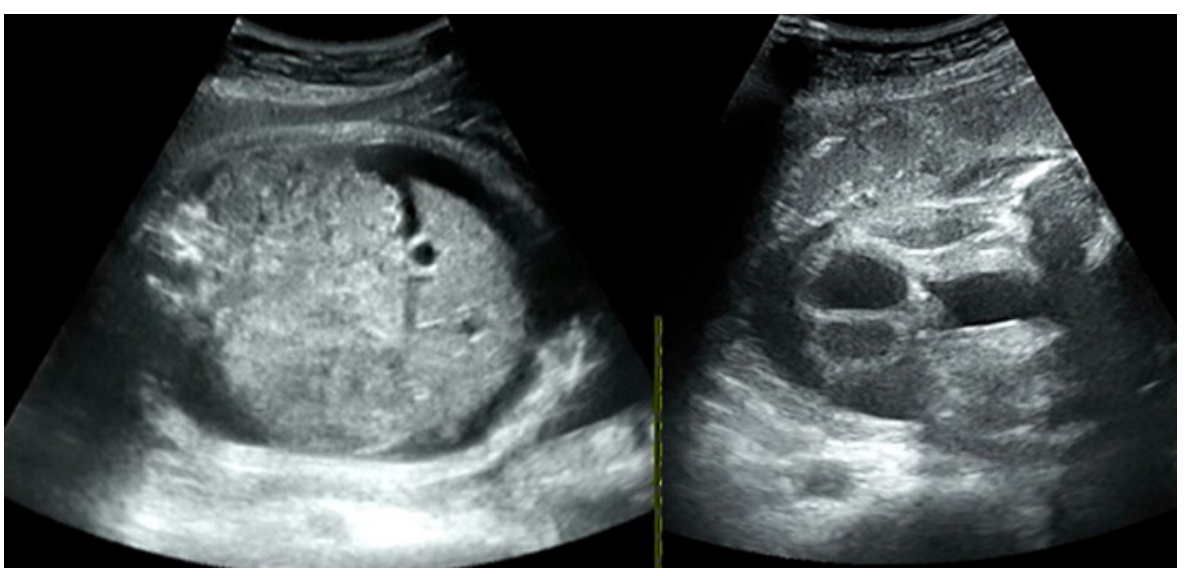

Fig. 1. Fetal ultrasonography at 31 weeks and 5 days of gestation showing a cystic lesion with ascites.

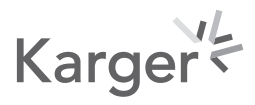



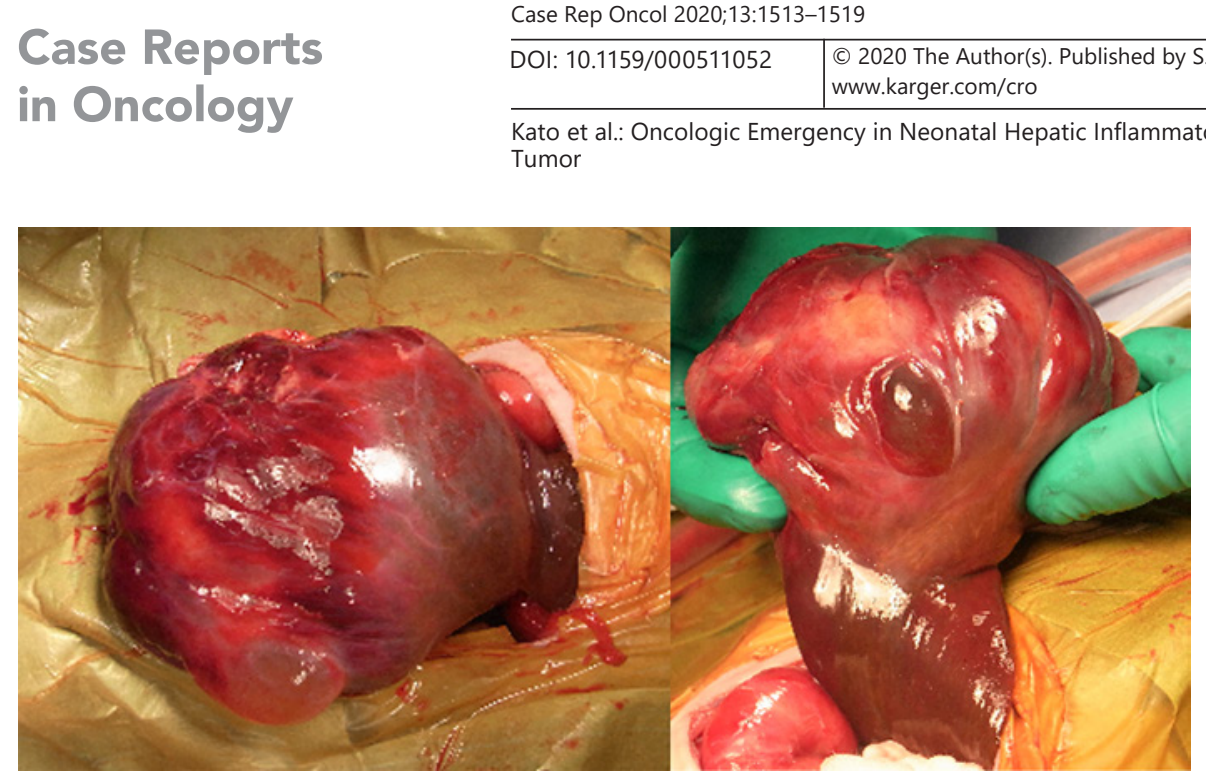

Fig. 2. The cystic mass arose from the left lateral segment of the liver.

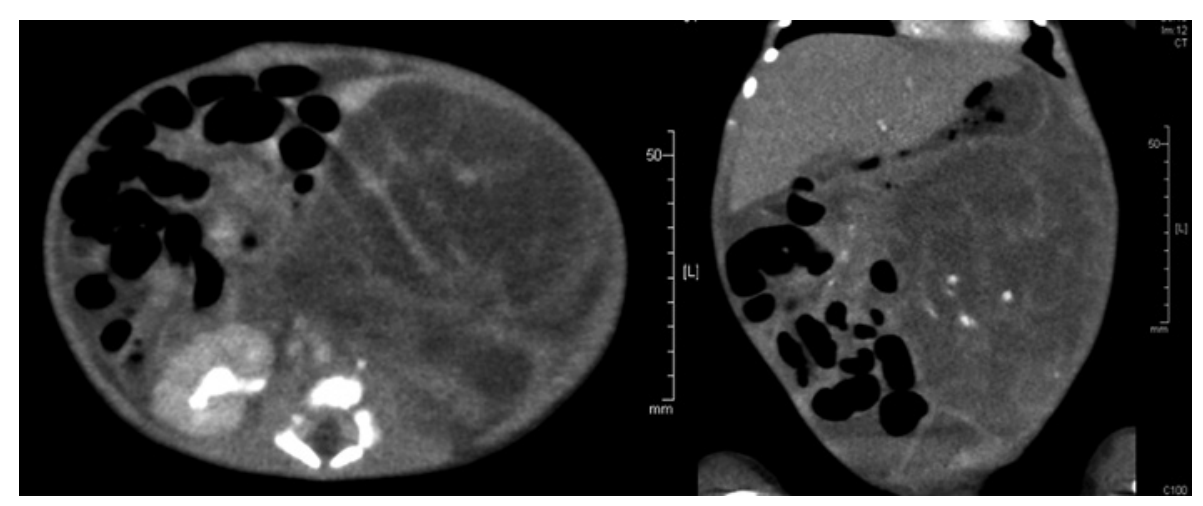

Fig. 3. Abdominal CT showed a hepatic tumor $(85 \times 62 \times 71 \mathrm{~mm})$ with a heterogeneously enhanced effect.

showed respiratory acidosis with pH 7.229, $\mathrm{PaCO}_{2} 60.1 \mathrm{~mm} \mathrm{Hg}, \mathrm{PaO}_{2} 61.9 \mathrm{~mm} \mathrm{Hg}, \mathrm{HCO}_{3} 24.2$ $\mathrm{mmol} / \mathrm{L}, \mathrm{BE}-3.4 \mathrm{mmol} / \mathrm{L}$, and Lac $14 \mathrm{mg} / \mathrm{dL}$. An emergency operation was performed on day 0 with the diagnosis of meconium peritonitis.

Abdominal computed tomography (CT) showed a hepatic tumor $(85 \times 62 \times 71 \mathrm{~mm})$ with an internal nonuniform contrast effect (Fig. 2).

Emergency surgery uncovered a large amount of ascites, which was removed as much as possible. Initially, the cystic lesion was thought to be an enlarged small intestine due to meconium peritonitis, but there were actually no obvious findings in the small intestine. Instead, the cystic mass arose from the left lateral segment of the liver (Fig. 3). The patient's breathing and circulation dynamics could be stabilized only by ascites removal, so only a tumor biopsy was performed.

Pathological findings by biopsy included spindle-shaped cell proliferation and infiltration of inflammatory cells, such as plasma cells and lymphocytes. Immunostaining showed positivity for desmin, vimentin, and $\alpha$-smooth muscle actin ( $\alpha \mathrm{SMA}$ ), but was negative for anaplastic lymphoma kinase, S-100, and myogenin. The patient was therefore diagnosed with IMT.

After the emergency surgery, the patient presented with redness of the abdominal wall due to spread of the inflammation. Prednisolone was administered for anti-inflammatory and tumor shrinkage effects. Tachypnea was alleviated, and blood gas analysis also reduced the respiratory acidosis with $\mathrm{pH}$ 7.457, $\mathrm{PaCO}_{2} 33.8 \mathrm{~mm} \mathrm{Hg}, \mathrm{PaO}_{2} 68.0 \mathrm{~mm} \mathrm{Hg}, \mathrm{HCO}_{3} 23.5 \mathrm{mmol} / \mathrm{L}$, 
Kato et al.: Oncologic Emergency in Neonatal Hepatic Inflammatory Myofibroblastic Tumor

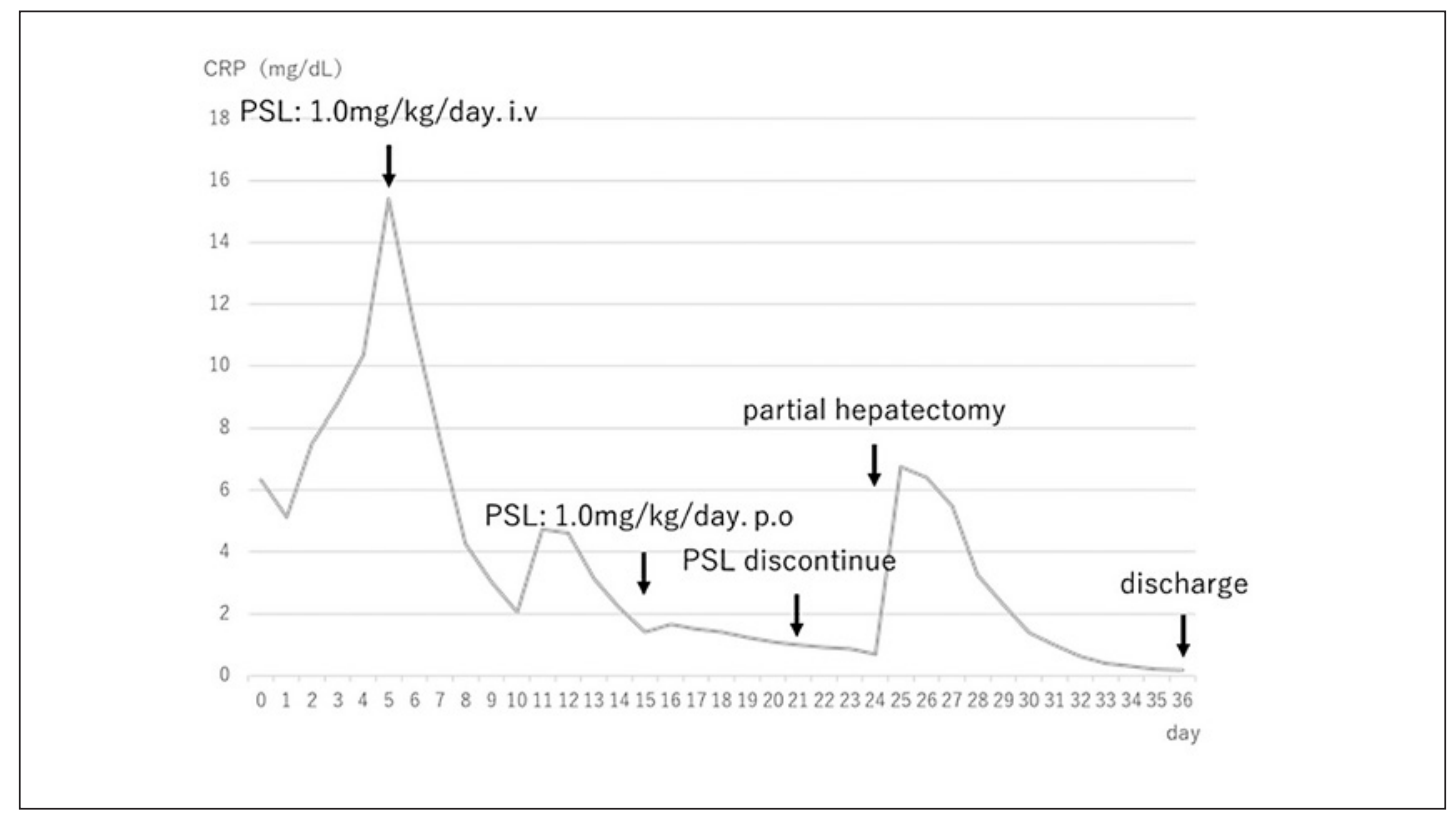

Fig. 4. CRP change from birth to discharge. PSL, prednisolone.

BE $0.7 \mathrm{mmol} / \mathrm{L}$, and Lac $12 \mathrm{mg} / \mathrm{dL}$. After medication with prednisolone, the blood test gradually showed a reduced inflammatory reaction (Fig. 4), but the tumor did not shrink in size, so we performed segment III partial hepatectomy on day 24 .

Intraoperative sonography showed no significant vascular infiltration or tumor embolism, and we performed segment IV partial hepatectomy. The hepatic IMT had fluid of multilocular and serous content, and protruded from the liver.

The tumor originating from the liver parenchyma showed uniform and atypical growth of inconspicuous spindle-shaped cells, and infiltration of main plasma cells, lymphocytes, blastoid cells, and eosinophil-like cells. Venous embolism and granulomatous changes due to thrombus were observed in the tumor, but there were no malignant findings, and negative malignancy at the surgical margin.

The postoperative course was uneventful and the patient was discharged on day 36 . Seven years after the operation there has been no recurrence or distant metastasis, and there have been no problems in growth and development.

\section{Discussion}

IMT is a solid tumor showing proliferation of the collagen matrix and spindle-shaped cells with proliferation of myofibroblasts and infiltration of inflammatory cells such as lymphocytes, plasma cells, and eosinophils. It rarely presents with recurrence or distant metastasis, so it is classified as a tumor with intermediate biological characteristics [1].

IMT originating from the liver is very rare; it was first reported by Pack and Baker in 1953 [2]. Hepatic IMT occurs predominantly in young adulthood, and there have been no previous reports of fetal or neonatal hepatic IMT. Moreover, only 1 case of IMT originating from the mesentery has been reported from the fetal period [3]. The etiology of IMT is not clearly understood, but it is possible that it is caused by inflammatory reactions such as infection, 
trauma, surgery, autoimmune diseases, vascular lesions, or chronic inflammation [4]. In particular, the inflammatory reaction in the digestive tract is reported to spread to the hepatic parenchyma to develop hepatic IMT $[5,6]$.

In the current case, fetal ultrasonography in the 31st week of gestation revealed a huge cystic lesion with ascites, but the patient's mother had no underlying disease and had an uneventful pregnancy. Transplacental infection was not suspected, because various antibodies were negative in the maternal blood test. The cause of IMT in this case is therefore unknown.

The chief causes of ascites in the fetal period are intrauterine infections (cytomegalovirus, toxoplasma, and syphilis), heart malformations, and metabolic diseases, among others. Some fetal ascites disappears naturally [7], but in this case, the cause of the ascites was thought to be a reaction to IMT. Fetal hydrops, the increase in fetal ascites, and the appearance of subcutaneous edema were therefore thought to become apparent because the fetus was not administered any medication during the fetal period. The child was delivered by emergency cesarean section. Hepatic IMT may be diagnosed from symptoms such as abdominal mass, hepatomegaly, or weight loss in infancy [6]. In this case, the patient presented an oncologic emergency due to respiratory failure after birth because the hepatic IMT was initially thought to be a huge cystic lesion rather than a tumor. Attention should therefore be paid to respiratory symptoms due to hepatic IMTs in the neonatal period.

Hepatic tumors with ascites can be distinguished in the fetal or neonatal period [8], but cases of oncologic emergency due to respiratory disorders in the neonatal period because of hepatic tumor and ascites are very rare. Abdominal paracentesis and ascites drainage are performed as emergency measures $[9,10]$. In this case, the cystic lesion was initially considered to be an enlargement of the small intestine from meconium peritonitis, so we elected to perform a laparotomy. The intraoperative findings revealed that the cystic lesion was a tumor originating from the liver. The patient's breathing and circulation dynamics could be stabilized only by removal of the ascites, so only a tumor biopsy was performed. Abdominal ultrasonography showed hepatic IMT with hypoechoic or hyperechoic images with the septum [11]. Plain CT shows hepatic IMT as a low-concentration tumor with unclear margins, while contrast-enhanced CT shows that the tumor has poorly defined peripheral enhancement in the arterial phase and a poorly defined periphery with an internal hypoattenuating area in the equilibrium phase with a low concentration inside [11]. In this case, ultrasonography showed a polycystic heterogeneous internal mass which was not typical of liver IMT, but CT showed that the tumor had a heterogeneous internal appearance, which was similar to that reported by Thompson et al. [3].

Diagnosis of hepatic IMT was confirmed by histopathological findings, but immunostaining analysis was used as an auxiliary diagnosis. In addition to representative vimentin, aSMA and muscle-specific actin, desmin, CD68 and pankeratin were shown to be positive [12]. The prognosis of hepatic IMT is generally good, since there have been reports of spontaneous regression [13] and improvement with nonsteroidal anti-inflammatory drugs or steroids for conservative treatment. Surgical resection is indicated when there are obstructions or exclusion symptoms that cause cholestasis or portal hypertension, or when the tumor does not shrink with conservative treatment [5]. In the current case, the pathological findings showed proliferation of spindle-shaped cells and infiltration of inflammatory cells such as plasma cells and lymphocytes, and, moreover, immunostaining analysis was positive for $\alpha \mathrm{SMA}$, muscle-specific actin, desmin and vimentin, and negative for anaplastic lymphoma kinase, S-100 and myogenin, which yielded the diagnosis of IMT.

Ascites redeposition was revealed in the postoperative course, and redness of the abdominal wall was presented due to inflammatory influence. Prednisolone was administered early after surgery for the purpose of an anti-inflammatory effect and tumor shrinkage,

\section{Karger'"}


which induced alleviation of the abdominal distention and tachypnea, and the blood tests also showed a reduced inflammatory response, so it was considered to beappropriate management for the oncologic emergency to remove the ascites by laparotomy and steroid treatment. There was no apparent shrinkage of the tumor, however; thus, surgical treatment was indicated. Partial hepatectomy was performed on day 24.

Hepatic IMT has a high risk of postoperative local recurrence in the case of incomplete tumor resection, a primary in the abdominal/pelvic cavity, or a tumor size $>8 \mathrm{~cm}[12,14]$. In addition, distant metastasis rarely occurs at the time of or within 1 year after the diagnosis, although in one report distant metastasis was diagnosed 9 years after the operation [15]. Seven years have passed since the currently reported patient's surgery, but long-term followup may be necessary with consideration of local recurrence or distant metastasis.

\section{Conclusion}

Huge hepatic IMTs can cause an oncologic emergency due to respiratory disorder during the neonatal period, requiring immediate management.

\section{Acknowledgements}

We acknowledge editing and proofreading by Benjamin Phillis from the Clinical Study Support Center at Wakayama Medical University.

\section{Statement of Ethics}

We have obtained written informed consent from the parents of the patient to publish this report.

\section{Conflict of Interest Statement}

The authors have no conflicts of interest to disclose.

\section{Funding Sources}

No funding was received.

\section{Author Contributions}

H.K. wrote the main text of the manuscript and collected photographs. Y.M. and H.Y. did the final revision of the text. All authors approved the final version of the manuscript.

\section{Karger'}




\section{References}

1 Fletcher CDM, Unni K, Mertens F. Pathology and genetics of tumours of soft tissue and bone. World Health Organization classification of tumours. Lyon: IARC Press; 2002.

2 Pack GT, Baker HW. Total right hepatic lobectomy; report of a case. Ann Surg. 1953 Aug;138(2):253.

3 Thompson RJ, Barrett AM, Dildey P. Congenital multifocal inflammatory pseudotumor: a case report. J Pediatr Surg. 2003 Oct;38(10):E17-9.

4 Sanders BM, West KW, Gingalewski C, Engum S, Davis M, Grosfeld JL. Inflammatory pseudotumor of the alimentary tract: clinical and surgical experience. J Pediatr Surg. 2001 Jan;36(1):169-73.

5 Thavamani A, Mandelia C, Anderson PM, Radhakrishnan K. Pediatric inflammatory myofibroblastic tumor of the liver: a rare cause of portal hypertension. ACG Case Rep J. 2019 Mar;27(6):1-4.

6 Al-Hussaini H, Azouz H, Abu-Zaid A. Hepatic inflammatory pseudotumor presenting in an 8-year-old boy: a case report and review of literature. World J Gastroenterol. 2015 Jul;28(21):8730-8.

7 Bavdekar A, Thakur N. Ascites in children. Indian J Pediatr. 2016 Nov;83(11):1334-40.

8 Isaacs H Jr. Fetal and neonatal hepatic tumors. J Pediatr Surg. 2007 Nov;42(11):1797-803.

9 Fukahori S, Asagiri K, Ishii S, Saikusa N, Hashizume N, Koga Y, et al. Role of the pediatric surgeon in pediatric oncologic emergencies - our experience and a review of 223 cases reported in the Japanese literature. Jap J Pediatr Hematol Oncol. 2014 Dec;51(5):499-506.

10 Giefer MJ, Murray KF, Colletti RB. Pathophysiology, diagnosis, and management of pediatric ascites. J Pediatr Gastroenterol Nutr. 2011 May;52(5):503-13.

11 Park JY, Choi MS, Lim YS, Park JW, Kim SU, Min YW, et al. Clinical features, image findings, and prognosis of inflammatory pseudotumor of the liver: a multicenter experience of 45 cases. Gut Liver. 2014 Jan;8(1):58-63.

12 Tang L, Lai EC, Cong WM, Li AJ, Fu SY, Pan ZY, et al. Inflammatory myofibroblastic tumor of the liver: a cohort study. World J Surg. 2010 Feb;34(2):309-13.

13 Dasgupta D, Guthrie A, McClean P, Davison S, Luntley J, Rajwal S, et al. Liver transplantation for a hilar inflammatory myofibroblastic tumor. Pediatr Transplant. 2004 Oct;8(5):517-21.

14 Coffin CM, Hornick JL, Fletcher CD. Inflammatory myofibroblastic tumor: comparison of clinicopathologic, histologic, and immunohistochemical features including ALK expression in atypical and aggressive cases. Am J Surg Pathol. 2007 Apr;31(4):509-20.

15 Morotti RA, Legman MD, Kerkar N, Pawel BR, Sanger WG, Coffin CM. Pediatric inflammatory myofibroblastic tumor with late metastasis to the lung: case report and review of the literature. Pediatr Dev Pathol. 2005 Mar; $8(2): 224-9$. 